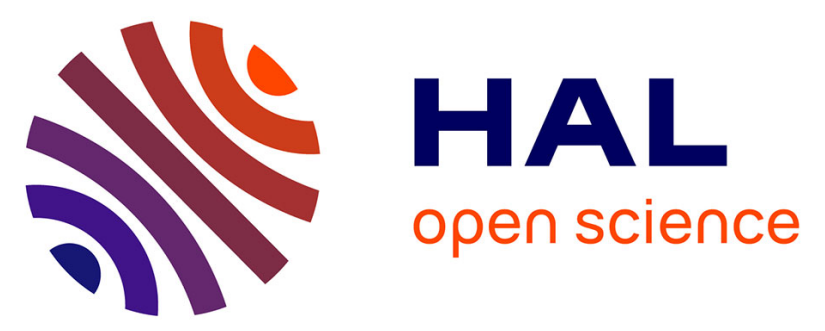

\title{
Etude par Microscopie Electronique et Mesures de Conductance Electrique In Situ de la Cristallisation de Couches a-Si Obtenues par Pyrolyse de Silane et Disilane par LPCVD dans des Conditions Ultra-Pures
}

\author{
T. Kretz, D. Pribat, P. Legagneux, F. Plais, O. Huet, M. Magis
}

\section{To cite this version:}

T. Kretz, D. Pribat, P. Legagneux, F. Plais, O. Huet, et al.. Etude par Microscopie Electronique et Mesures de Conductance Electrique In Situ de la Cristallisation de Couches a-Si Obtenues par Pyrolyse de Silane et Disilane par LPCVD dans des Conditions Ultra-Pures. J. Phys. IV, 1995, 05 (C3), pp.C3-291-C3-296. 10.1051/jp4:1995329 • jpa-00253695

\section{HAL Id: jpa-00253695 https://hal.science/jpa-00253695}

Submitted on 1 Jan 1995

HAL is a multi-disciplinary open access archive for the deposit and dissemination of scientific research documents, whether they are published or not. The documents may come from teaching and research institutions in France or abroad, or from public or private research centers.
L'archive ouverte pluridisciplinaire HAL, est destinée au dépôt et à la diffusion de documents scientifiques de niveau recherche, publiés ou non, émanant des établissements d'enseignement et de recherche français ou étrangers, des laboratoires publics ou privés. 


\title{
Etude par Microscopie Electronique et Mesures de Conductance Electrique In Situ de la Cristallisation de Couches a-Si Obtenues par Pyrolyse de Silane et Disilane par LPCVD dans des Conditions Ultra-Pures
}

\author{
T. Kretz, D. Pribat, P. Legagneux, F. Plais, O. Huet et M. Magis \\ Thomson-CSF LCR, Domaine de Corbeville, 91404 Orsay cedex, France
}

\begin{abstract}
Résumé. Des couches de silicium amorphe de haute pureté ont été réalisées dans un réacteur de type LPCVD (Low Pressure Chemical Vapour Deposition) dans des conditions ultra-pures à partir des gaz silane et disilane. L'évolution de la densité de germes par unité de surface est suivie à $580^{\circ} \mathrm{C}$ par microscopie électronique en cours de cristallisation en phase solide, ce qui permet d'en déduire la vitesse de germination à cette température. D'autre part, la variation de la taille des grains, sur les couches complètement cristallisées, est étudiée par microscopie électronique à transmission (MET) en fonction de la température de recuit. La fraction volumique cristallisée des films amorphes en cours de recuits isothermes est suivie par des mesures de conductance électrique in situ. Par combinaison de ces deux méthodes de caractérisation nous pouvons remonter avec précision aux paramètres thermodynamiques de la cristallisation concernant les deux types de dépôt.
\end{abstract}

\begin{abstract}
High purity amorphous silicon layers were deposited by low pressure chemical vapour deposition (LPCVD) from either silane or disilane gases. During the solid phase crystallisation of the amorphous layers at $580^{\circ} \mathrm{C}$, the grain density was followed by electron microscopy and the nucleation rate at that temperature was extracted. Moreover, the variation of the grain size of the completly cristallised films as a function of annealing temperature was studied by transmission electron microscopy (TEM). The crystalline fraction was monitored by in situ electrical conductance measurements during isothermal annealings. Using these two caracterisation methods, we were able to precisely determine the thermodynamic cristallisation parameters for both types of film studied.
\end{abstract}

\section{INTRODUCTION}

De nombreuses études ont été effectuées ces dernières années sur les couches minces de silicium polycristallin (poly-Si). Les porteurs dans ce matériau présentent une mobilité de un à quatre ordres de grandeur supérieure à celle du silicium amorphe hydrogéné (a-Si:H). Le poly-Si est donc surtout étudié en vue de son application à la fabrication de transistors en couches minces pour les écrans plats à cristaux liquides à matrices actives comprenant un transistor de commande par point image [1]. En particulier, il permet l'intégration directe des circuits périphériques de commande de la matrice active sur le même support, ce qui simplifie la fabrication par rapport anx matrices utilisant le a-Si:H dans lesquelles l'électronique de commande en silicium monocristallin doit être rapportée sous forme discrète à la périphérie de la plaque de verre.

Beaucoup d'efforts ont été dévolus à l'obtention d'un matériau polycristallin présentant des gros grains. La méthode généralement utilisée consiste à cristalliser thermiquement à basse température $\left(\leq 600^{\circ} \mathrm{C}\right)$ le a-Si déposé par décomposition pyrolytique du gaz silane $\left(\mathrm{SiH}_{4}\right)$ aux alentours de $550^{\circ} \mathrm{C}$ [2]. Néanmoins, sans l'intervention de procédures post-dépôt très sophistiquées $[3,4]$ la taille des grains reste faible $(<500 \mathrm{~nm})$. D'après de récentes études sur les dépôts amorphes à base de gaz disilane $\left(\mathrm{Si}_{2} \mathrm{H}_{6}\right)$ [5-8], des grains de quelques microns peuvent être directement obtenus par cristallisation thermique à basse température. Cependant, concernant ces dernières couches, peu d'informations ont été données sur les paramètres thermodynamiques de la cristallisation.

Nous présentons dans ce travail l'étude de la cristallisation en phase solide de silicium amorphe de haute pureté déposé par pyrolyse des gaz silane et disilane par LPCVD. L'évolution de la densité de germes au 
cours du temps de recuit a été suivie tout d'abord par microscopie électronique à balayage (MEB). Nous avons ensuite déterminé les paramètres de la cristallisation tels que les énergies d'activation de la vitesse de croissance, $\mathrm{E}_{\mathrm{g}}$, et du taux de nucléation, $\mathrm{E}_{\mathrm{n}}$, pour les couches à base de $\mathrm{SiH}_{4}$ et de $\mathrm{Si}_{2} \mathrm{H}_{6}$ à partir de mesures de conductance électrique in situ et de la variation de la taille des grains en fonction de la température de recuit.

\section{DEPOT DU SILICIUM AMORPHE}

Le réacteur UHVCVD utilisé [9] pour déposer les couches de silicium amorphe se distingue des réacteurs LPCVD classiques à murs chauds par la présence d'un sas de chärgement qui permet de maintenir constamment le tube de dépôt sous vide. De plus, il est équipé d'un système de pompage ultravide dont une pompe primaire sèche et une pompe secondaire turbomoléculaire à paliers magnétiques. Pour une température de l'ordre de $550^{\circ} \mathrm{C}$, le vide résiduel dans le réacteur après quelques cycles de dépôt est de $2.10^{-9}$ Torr dont $90 \%$ d'hydrogène. La pression totale des gaz résiduels $\left(\mathrm{H}_{2} \mathrm{O}, \mathrm{CO}, \mathrm{CO}_{2}\right.$ et $\left.\mathrm{O}_{2}\right)$ est inférieure à $2.10^{-10}$ Torr. Les gaz de process à fort débit $\left(\mathrm{H}_{2}, \mathrm{SiH}_{4}\right)$ ont été purifiés à l'aide de résines organiques de type Nanochem qui adsorbent les polluants. Pour le disilane, qu'on utilise qu'à faible débit, des getters sont employés. Ce système d'épuration permet de limiter le taux de chaque impureté $\left(\mathrm{H}_{2} \mathrm{O}, \mathrm{CO}, \mathrm{CO}_{2}\right.$ et $\left.\mathrm{O}_{2}\right)$ dans les gaz process à environ $10 \mathrm{ppb}$.

Les couches de a-Si, d'épaisseur $80 \mathrm{~nm}$, étudiées dans ce travail ont été déposées sur des substrats de silicium monocristallin oxydés thermiquement. Les conditions de dépôt sont présentées dans le tableau 1. La pression et la vitesse de dépôt sont ajustées respectivement à 2,6 nm.min ${ }^{-1}$ et 2 Torr dans les deux cas de figure considérés.

Tableau 1 : Conditions de dépôt des couches a-Si.

\begin{tabular}{|c|c|c|c|}
\hline $\mathrm{SiH}_{4}[\mathrm{sccm}]$ & $\mathrm{Si}_{2} \mathrm{H}_{6}[\mathrm{sccm}]$ & $\mathrm{H}_{2}[\mathrm{sccm}]$ & Temperature $\left[{ }^{\circ} \mathrm{C}\right]$ \\
\hline 500 & 0 & 1500 & 550 \\
\hline 0 & 40 & 1960 & 480 \\
\hline
\end{tabular}

Les analyses SIMS effectuées sur des couches amorphes déposées sur des substrats de Si de type FZ donnent, par comparaison avec le substrat, des taux d'impuretés en oxygène et carbone très faibles. Par exemple, des concentrations d'oxygène de quelques $10^{17} \mathrm{~cm}^{-3}$ pour le dépôt à base de silane et de quelques $10^{16} \mathrm{~cm}^{-3}$ pour le disilane sont obtenues [9]. Ces taux sont plus de deux ordres de grandeurs plus faibles que ceux rapportés dans la littérature pour des dépôts LPCVD $[8,10]$.

\section{ETUDE DE LA CRISTALlisation EN PHASE SOLIDE}

\subsection{Etude de la germination à $580^{\circ} \mathrm{C}$}

Au fur et à mesure de l'avancement de la cristallisation, la cinétique de germination et de croissance des grains est suivie au MEB, après révélation chimique (Secco etch [11]) de la phase amorphe et des joints de grains. Nous présentons sur la fig. 1 les photographies obtenues sur une couche déposée amorphe à partir du gaz silane, après recuits a $580^{\circ} \mathrm{C}$ pendant $20,30,40$ et 50 minutes. Après 20 minutes de recuit la cristallisation a débuté et les premiers germes stables, de forme circulaire, sont visibles. Ils apparaissent comme noyés dans la phase amorphe majoritaire (fig.1.a). D'après J.Morgiel et al. [12], lorsque la nucléation débute à l'interface couche-substrat, les germes stables sont déjà maclés intérieurement à l'état initial et évoluent indépendamment les uns des autres mais plus rapidement suivant la direction de maclage. Cette progression plus rapide suivant une direction donnée explique la forme allongée finale des grains. Pendant que les cristallites évoluent, d'autres embryons apparaissent et dépassent la taille critique (fig.1.b) pour évoluer suivant un cycle de croissance identique à leurs prédécesseurs. L'augmentation de la densité et de la taille des cristallites en cours de recuit est bien mise en évidence sur les différentes photographies. Au bout de 40 minutes de recuit (fig.1.c), on observe que quelques cristallites finissent par se rencontrer. Au point de contact le front de croissance des grains est donc stopé et un joint de grain se forme. Sur des observations en coupe transverse au MET nous avons pu constater que la germination débute à l'interface couche-substrat de façon hétérogène [13]. Ce mécanisme de nucléation est en accord avec les résultats publiés dans la littérature [12,14]. Ainsi, dès que les germes stables sont formés à l'interface inférieure, ils croissent d'une façon tridimensionnelle en atteignant rapidement la surface de la couche, puisque cette dernière est relativement mince (environ $80 \mathrm{~nm}$ ), pour s'étendre ensuite latéralement par croissance 
bidimensionnelle. Après 50 minutes de cristallisation, la majorité des grains se rencontrent si bien qu'un quasi continuum de cristallites adjacentes peut être observé sur la totalité du champ de la photographie.

La densité de germes par unité de surface mesurée a $580^{\circ} \mathrm{C}$ en fonction de la durée de recuit est représentée sur la fig.2. Elle est déterminée par comptage des cristallites visibles sur les différentes photographies en vue plane prises au MEB, après révélation de la phase amorphe, à différents intervalles de cristallisation. On peut y observer trois régimes distincts identiques à ceux mentionnés par Christian [16] lors de la description de la cinétique classique de germination, soit : un régime d'incubation au dessous duquel aucune germination apparente n'est détectable, un régime transitoire et un régime de germination constante durant lequel le nombre de cristallites augmente linéairement en fonction de la durée de recuit. A noter qu'un régime de saturation ne peut être mis en évidence par cette technique, dans la mesure où le comptage des grains après jonction est peu précis sinon ambigu.

Après une courte durée de nucléation transitoire, le régime de germination constante s'établit rapidement. L'hypothèse formulée dans la théorie classique de cristallisation qui considére le taux de nucléation comme constant peut donc être admise. Le taux de germination à la température de $580^{\circ} \mathrm{C}$, déterminé dans la région linéaire de la fig. 2, est de l'ordre de $7,6.10^{7} \mathrm{~cm}^{-2} \mathrm{~min}^{-1}$ dans le cas de la couche à base de silane.

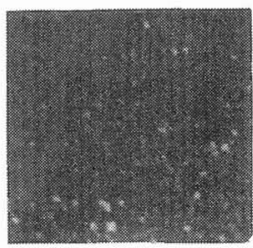

(a) $20 \mathrm{~min}$

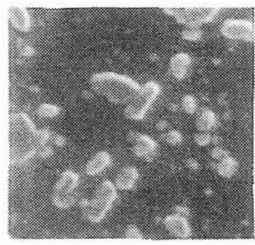

(c) $40 \mathrm{~min}$

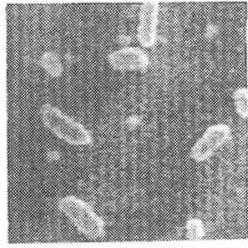

(b) $30 \mathrm{~min}$

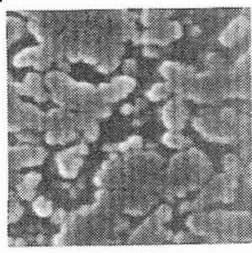

(d) $50 \mathrm{~min}$

Fig.1 : Vues au MEB après révélation Secco de la couche amorphe à base de silane recuite à $580^{\circ} \mathrm{C}$ durant différentes durées.

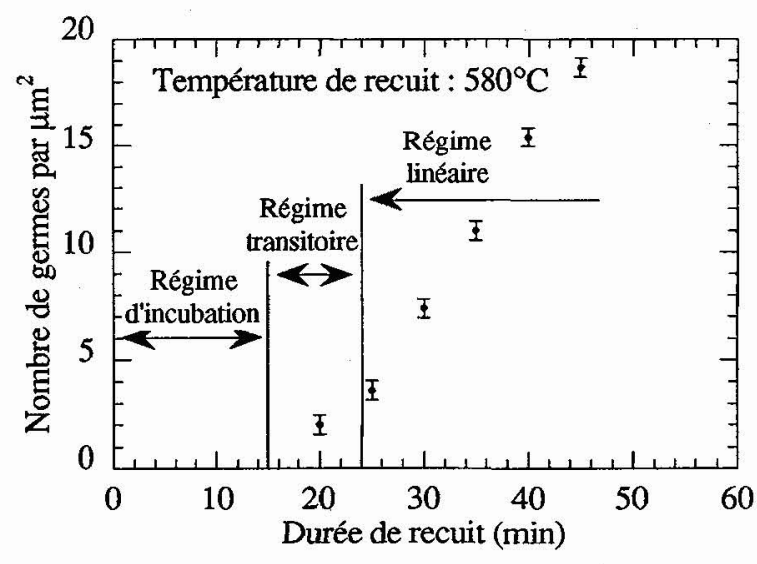

Fig. 2 : Variation du nombre de germes par unité de surface en fonction de la durée de cristallisation de la couche à base de silane recuite à $580^{\circ} \mathrm{C}$.

Une étude similaire a été effectuée dans le cas des couches à base de disilane et un processus de cristallisation identique a été observé. La nucléation hétérogène s'amorce au niveau de l'interface couchesubstrat et les germes atteignent rapidement la surface de la couche pour croître ensuite latéralement. Un taux de nucléation de l'ordre de $2,2.10^{5} \mathrm{~cm}^{-2} \mathrm{~min}^{-1}$ a été trouvé à $580^{\circ} \mathrm{C}$, valeur environ 100 fois plus faible que celle obtenue pour le silane.

\subsection{Mesure de la taille de grains en fonction de la température de recuit}

La taille des grains est déterminée par MET en vue plane en champ sombre en mesurant l'axe principal des cristallites dans les couches complètement cristallisées [13,17]. Une "taille caractéristique" est obtenue en moyennant les mesures relevées sur une trentaine des plus gros grains. Un écart de taille de grains de plus d'un facteur dix est mis en évidence entre les couches à base de silane et celles à base de disilane [17].

Dans le cadre du modèle simple développé par M.K.Hatalis et D.W.Greve [2], on montre que la taille des cristallites varie en fonction inverse de la température de recuit de cristallisation suivant une loi exponentielle, dont la pente représente l'énergie caractéristique de la taille des grains, $E_{S}=\left(E_{n}-E_{g}\right) / 3$ $[13,17]$. Sur la fig.3, on a porté la taille des cristallites obtenues après des recuits isothermes effectués à des températures comprises entre 540 et $600^{\circ} \mathrm{C}$ sur des couches à base de silane ou de disilane. On remarque que l'allure des courbes est bien celle prévue par l'équation d'Hatalis-Greve. Les énergies d'activation de la taille des grains, $E_{S}$, sont de 0,20 et $0,83 \mathrm{eV}$ pour les couches réalisées respectivement à base de silane et de disilane. Ces valeurs sont en bon accord avec celles obtenues par Hatalis et Greve [2] et Iverson et Reif [3] pour des matériaux à petits et gros grains respectivement. 


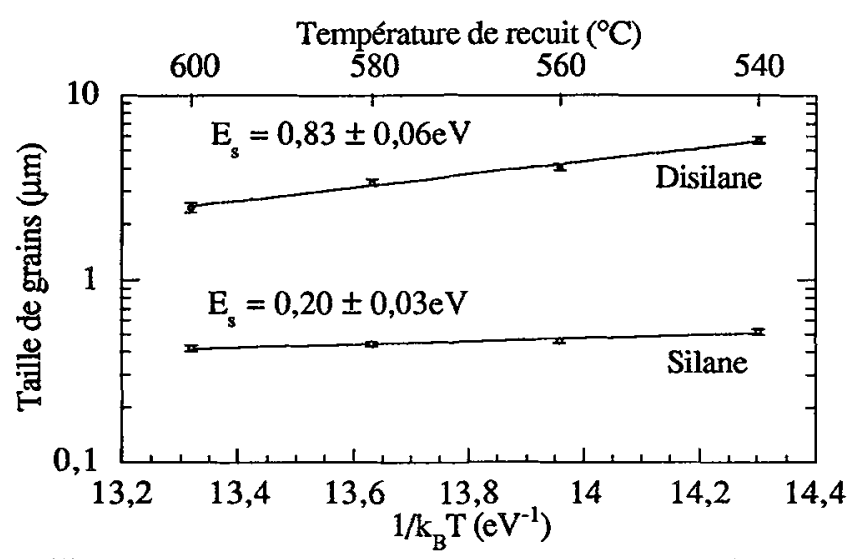

Fig.3 : Variation de la taille des grains en fonction de $1 / \mathrm{k}_{\mathrm{B}} \mathrm{T}$ pour les dépôts à base de silane et de disilane.

\subsection{Evaluation du temps caractéristique de cristallisation par mesure de la conductance électrique in situ}

Deux contacts de platine de diamètre $1,5 \mathrm{~mm}$ ont été évaporés au canon à électrons à la surface des couches amorphes à analyser. Les variations de courant sont déterminées par mesure classique entre deux pointes, en cours de recuit, en appliquant à la sortie de la source de tension d'un électromètre une valeur faible de l'ordre de $0,4 \mathrm{~V}$. Pour chaque point enrégistré, nous avons contrôlé le comportement ohmique de la résistance des contacts et de la couche entre -10 et $+10 \mathrm{~V}$. Un thermocouple $\mathrm{Pt} / \mathrm{Pt}-\mathrm{Rh} 10 \%$ placé près de l'échantillon permet de suivre en permanence la température du four. Le principe de la méthode est détaillée dans la référence 13.

Sur la fig.4, nous avons représenté l'évolution typique de la conductance électrique en fonction de la durée de recuit dans le cas du silane pour différentes températures de cristallisation, à savoir, 540, 560, 580 et $600^{\circ} \mathrm{C}$; une évolution identique est observée dans le cas des couches à base de disilane. Précisons pour ces dernières que les températures de recuit ont été fixées entre 600 et $630^{\circ} \mathrm{C}$ afin de s'affranchir d'un temps d'incubation et d'une durée de cristallisation complète trop longs. La conductance est normalisée par rapport à la conductance de la phase amorphe, $G_{a}$, afin d'éliminer toute ambiguité due à la forme géométrique des échantillons. Si l'on prend en compte les concentrations des phases amorphe et polycristalline présentes dans la couche, ainsi que leur conductivité respective, il est possible de relier les courbes de conductance aux différents régimes de germination décrits dans le paragraphe 3.1. Au début de la cristallisation des grains isolés (ou des amas de grains) existant dans la couche sont noyés dans un milieu amorphe peu conducteur, il s'ensuit que, la conductance globale du mélange amorphe-polycristal évolue faiblement. Par contre, dès qu'un chemin continu de grains se forme entre les deux électrodes de mesures, la conductance de la couche augmente brusquement et le seuil de percolation est atteint [18-20]. D'après J.P.Clerc et al. [20], la fraction volumique cristallisée critique, $X_{c}$, atteinte au temps tperc, est donnée en croissance bidimensionnelle par la relation suivante : $G\left(t_{\text {perc }}\right) / G_{a}=\sqrt{G_{c}} / G_{a}$, où $G c$ représentent la conductance relevée en fin de recuit après cristallisation complète des couches. Dans ces conditions, d'après l'expression d'Avrami-Mehl-Johnson [21,22] de la fraction volumique cristallisée pour une croissance à deux dimensions et de l'équation de Landauer [23] de la conductance moyenne d'un mélange binaire à deux phases de conductances respectives $\mathrm{G}_{\mathrm{a}}$ et $\mathrm{G}_{\mathrm{c}}$ aléatoirement réparties, on se trouve au seuil de percolation lorsque environ $40 \%$ de la couche amorphe est cristallisée. Ce résultat est en accord avec la littérature. Lorsque la couche est entièrement cristallisée, la région de saturation des courbes de conductance est atteinte (fig.4). On constate que la conductance $\mathrm{G}_{\mathrm{c}}$ de la couche entièrement cristallisée, le temps d'incubation ainsi que la durée de la cristallisation complète des couches croissent en fonction de l'inverse de la température de recuit. Cette évolution peut-être rapprochée de l'augmentation de la taille des grains lorsque la température de recuit diminue (voir paragraphe précédent et [2]).

Afin d'extraire des mesures de conductance les informations plus précises sur le temps caractéristique de cristallisation $\left(t_{c}\right)$, nous avons combiné l'expression d'Avrami-Mehl-Johnson de la fraction volumique cristallisée [21,22] avec l'équation de Landauer [23] et ajusté ce modèle théorique aux points expérimentaux. Cette procédure est détaillée dans les références 13 et 17 . Sur la figure 5 , on a tracé le temps caractéristique de cristallisation $t_{c}$ en fonction de $1 / \mathrm{k}_{\mathrm{B}} \mathrm{T}$ pour les deux types de dépôts. Nous constatons que l'inverse du temps caractéristique de cristallisation suit une loi d'Arrhénius dont il est aisé 
d'extraire la pente qui représente l'énergie caractéristique du temps de cristallisation, $\mathrm{E}_{\mathrm{tc}}$. Pour une croissance à deux dimensions, $E_{t c}$ est reliée à $E_{n}$ et $E_{g}$ par la relation : $E_{t c}=\left(E_{n}+2 E_{g}\right) / 3[13,17]$. Nous avons ainsi obtenu des énergies de l'ordre de $2,87 \mathrm{eV}$ et de $3,12 \mathrm{eV}$ pour les couches déposées à base respectivement de silane et de disilane.

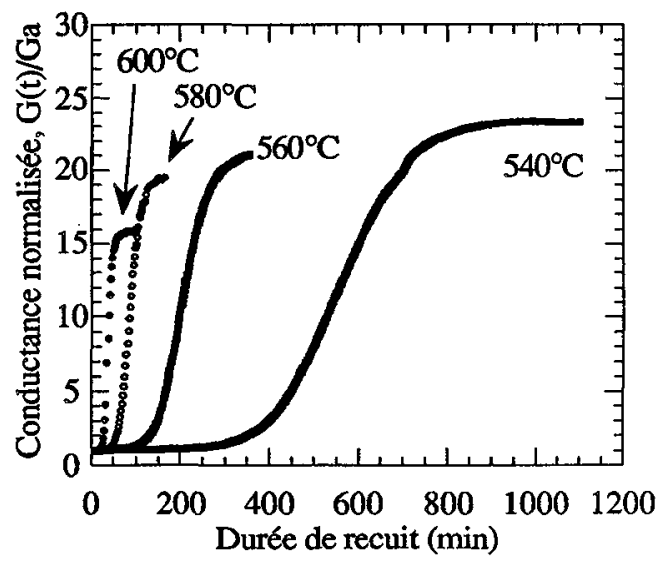

Fig.4 : Variation de la conductance en fonction de la durée de recuit pour différentes températures de cristallisation de la couche amorphe déposée par décomposition thermique de $\mathrm{SiH}_{4}$.

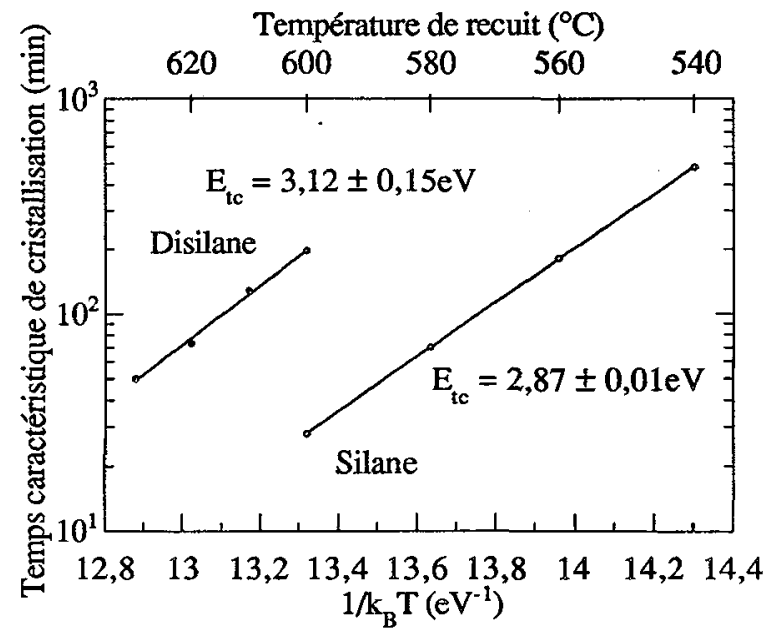

Fig.5 : Temps caractéristique de cristallisation des dépôts obtenus à partir de silane et de disilane en fonction de $1 / \mathrm{k}_{\mathrm{B}} \mathrm{T}$ pour différentes températures de recuit.

\subsection{Energies d'activation du taux nucléation et de la vitesse de croissance}

A partir des énergies caractéristiques de la taille des grains, $\mathrm{E}_{\mathrm{S}}$, et du temps caractéristique de cristallisation, $E_{t c}$, nous pouvons remonter aux énergies d'activation de nucléation, $E_{n}$, et de croissance, $E_{g}$, pour chaque dépôt. Ces dernières peuvent être directement reliées aux paramètres thermodynamiques, tels que l'enthalpie maximale de formation du germe de taille critique, $\Delta \mathrm{H}^{*}$, et l'enthalpie libre de passage de la phase amorphe vers la phase cristalline, $\Delta \mathrm{h}_{\mathrm{m}}$, paramètres qui décrivent phénoménologiquement la croissance cristalline des couches amorphes [16]. Les résultats sont rassemblés dans le tableau 2:

Tableau 2: Paramètres de cristallisation pour les couches à base de silane et de disilane.

\begin{tabular}{|c|c|c|c|c|c|}
\hline & $\mathrm{E}_{\mathrm{tc}}[\mathrm{eV}]$ & $\mathrm{E}_{\mathrm{S}}[\mathrm{eV}]$ & $\mathrm{E}_{\mathrm{n}}[\mathrm{eV}]$ & $\mathrm{E}_{\mathrm{g}}=\Delta \mathrm{h}_{\mathrm{m}}[\mathrm{eV}]$ & $\Delta \mathrm{H}^{*}[\mathrm{eV}]$ \\
\hline $\mathrm{SiH}_{4}$ & $2,87 \pm 0,01$ & $0,20 \pm 0,03$ & $3,27 \pm 0,06$ & $2,67 \pm 0,03$ & $0,60 \pm 0,07$ \\
\hline $\mathrm{Si}_{2} \mathrm{H}_{6}$ & $3,12 \pm 0,15$ & $0,83 \pm 0,06$ & $4,78 \pm 0,19$ & $2,29 \pm 0,16$ & $2,49 \pm 0,25$ \\
\hline
\end{tabular}

Remarquons qu'à partir de la vitesse de germination déterminée dans le paragraphe 3.1 , pour une température de recuit donnée $\left(580^{\circ} \mathrm{C}\right)$ et l'énergie d'activation correspondante (tab.2) nous pouvons remonter à l'expression globale du taux de germination pour les dépôts à base de silane et de disilane [13].

\section{DISCUSSION}

D'une façon générale, on note une augmentation appréciable de l'énergie d'activation de germination $E_{n}$ lorsqu'on passe du dépôt à base de silane au dépôt à base de disilane. Ceci peut s'expliquer par l'augmentation du désordre dans les couches lorsque la température de dépôt diminue $[2,6,7,13]$. En effet, lorsque la température de dépôt est de l'ordre de $550^{\circ} \mathrm{C}$, les atomes de silicium peuvent s'associer en cours de dépôt pour former des régions où l'arrangement cristallin est proche de l'arrangement tétraédrique parfait du silicium monocristallin. Ces régions sont localisées près de l'interface couche-substrat puisque cette partie de la couche est la plus longtemps confrontée à la température de dépôt. Ainsi ces zones, plus ou moins ordonnées, sont des sites qui, durant le recuit de cristallisation, favoriseront l'augmentation du taux 
de germination ce qui explique probablement la nucléation préférentielle à l'interface a-Si/substrat. Inversement, lorsque la température de dépôt est diminuée, le désordre présent dans la couche amorphe augmente et la formation de germes de taille critique est donc plus difficile; le temps d'incubation s'en trouve augmenté. Le nombre de germes apparaissant par unité de surface est diminué et la taille finale des grains après cristallisation complète des couches est augmentée.

Les faibles concentrations d'impuretés dans les couches analysées peuvent expliquer les faibles valeurs des énergies d'activation de croissance déterminées dans ce travail [24]. En effet, pour des dépôts réalisés dans des réacteurs LPCVD classiques $[3,4,14]$ où la concentration d'oxygène est de plus de deux ordres de grandeur plus importante [10] les valeurs de $\mathrm{E}_{\mathrm{g}}$ sont supérieures à la nôtre. Tandis que pour des dépôts réalisés sous ultra-vide par évaporation sous faisceau d'électrons $[25,26]$ sur des substrats à base de silice, les énergies d'activation sont comparables à nos résultats.

\section{CONCLUSIONS}

La cinétique de cristallisation de couches amorphes de silicium de haute pureté a été étudiée. Les couches ont été déposées par décomposition thermique des gaz silane et disilane à une pression de 2 Torr aux températures respectivement de $550^{\circ} \mathrm{C}$ et $480^{\circ} \mathrm{C}$. La vitesse de dépôt était de l'ordre de $2,6 \mathrm{~nm} \cdot \mathrm{min}^{-1}$ dans les deux cas considérés. Les énergies d'activation de la vitesse de croissance et du taux de nucléation ont été extraites par ajustement des mesures de conductances électriques in situ effectués à différentes températures et des observations au TEM des couches complètement cristallisées. Les paramètres thermodynamiques de la thérie classique de la cristallisation en sont déduits. L'enthalpie maximale de formation du germe de taille critique est de l'ordre de $0,60 \mathrm{eV}$ pour le silane et $2,49 \mathrm{eV}$ pour le disilane. L'enthalpie libre de passage de la phase amorphe vers la phase cristalline est de l'ordre de $2,67 \mathrm{eV}$ pour le silane et $2,29 \mathrm{eV}$ pour le disilane. Ces différentes énergies correspondent à un taux de nucléation plus faible et une vitesse de croissance plus importante dans le cas du disilane. Pour cette raison, la taille des grains est nettement plus importante pour ce dernier par rapport aux couches à base de silane.

\section{REFERENCES}

[1] O'Mara W.C., Solid State Technology 34 décembre (1991) 65.

[2] Hatalis M.K. et Greve D.W., J. Appl. Phys. 63 (1988) 2260.

[3] Iverson R.B. et Reif R., J. Appl. Phys. 62 (1987) 1675.

[4] Wu I.W., Chiang A., Fuse M., Öveçoglu L. et Huang T.Y., J. Appl. Phys. 65 (1989) 4036.

[5] Scheid E., De Mauduit B., Taurines P. et Bielle-Daspet D., Jpn. J. Appl. Phys. 29 (1990) L2105.

[6] Nakazawa K., J. Appl. Phys. 69 (1991) 1703.

[7] Hong C.H., Park C.Y. et Kim H.J., J. Appl, Phys. 71 (1992) 5427.

[8] Voutsas A.T. et Hatalis M.K., J. Electrochem. Soc. 140 (1993) 871.

[9] Pribat D., Plais F., Legagneux P., Kretz T., Stroh R., Huet O., Walaine C., Magis M., Jiang N., Hugon M.C. et Agius B., Rev. Techn. Thomson-CSF 26 (1994) 73.

[10] Kamins T.I. et Turner J.E., Solid State Technology 33 April (1990) 80.

[11] Secco d'Aragona F., J. Electrochem. Soc. 119 (1972) 948.

[12] Morgiel J., Wu I.W., Chiang A. et Sinclair R., Mat. Res. Soc. Symp. Proc. 182 (1990) 191.

[13] Kretz T., Thèse, Université Louis Pasteur de Strasbourg I, ${ }^{\circ}$ d'ordre 1662 (1993).

[14] Bisaro R., Magariño J., Zellama K., Squelard S., Germain P. et Morhange J.F., Phys. Rew. $B 31$ (1985) 3568.

[15] Kobayashi K., Nijs J. et Mertens R., J. Appl. Phys. 65 (1989) 2541.

[16] Christian J.W., Physical Metallurgy (R.W.Cahn, Amsterdam, 1970), Chapter 10, p.471.

[17] Kretz T., Stroh R., Legagneux P., Huet O., Magis M. et Pribat D., "Polycrystalline semiconductors III - Physics and Technology", Solid State Phenomena Vol. XXX, éd. par Strunk H.P., Werner J.H., Fortin B. et Bonnaud O. (Trans. Tech., Zürich, 1994) en cours d'édition.

[18] de Gennes P.G., La Recherche $n^{\circ} 72$ vol.7 (1976) 919.

[19] Ottavi H., Clerc J., Giraud G., Roussenq J., Guyon E. et Mitescu C.D., J. Phys. C : Solid State Phys. 11 (1978) 1311.

[20] Clerc J.P., Giraud G., Laugier J.M. et Luck J.M., Adv. in Phys. 39 (1990) 191.

[21] Avrami M., J. Chem. Phys. 7 (1939) 1103.

[22] Johnson W.A. et Mehl R.F., Trans. Am. Inst. Min. Metall. Pet. Eng. 135 (1939) 416.

[23] Landauer R., J. Appl. Phys. 23 (1952) 779.

[24] Kennedy E.F., Csepregi L. et Mayer J.W., J. Appl. Phys. 48 (1977) 4241.

[25] Olson G.L. et Roth J.A., Materials Science Reports 3 (1988) 1.

[26] . Zellama K., Germain P., Squelard S., Bourgoin J.C. et Thomas P.A., J. Appl. Phys. 50 (1979) 6995. 